Tohoku J. Exp. Med., 2007, 212, 107-113

\title{
Impact of Glucose Intolerance on Coronary Calcified Lesions Evaluated Using Multislice Computed Tomography
}

\author{
Kyoko Nomura, ${ }^{1}$ Toshikazu Yamanouchi, ${ }^{2}$ Gwang U Kim, ${ }^{3}$ Kazuhiro Ohwaki ${ }^{1}$ \\ and EIJI YANO ${ }^{1}$ \\ ${ }^{1}$ Department of Hygiene and Public Health, School of Medicine, Teikyo University, Tokyo, Japan \\ ${ }^{2}$ Department of Internal Medicine, Teikyo University Hospital, Tokyo, Japan \\ ${ }^{3}$ Devision of Internal Medicine, Nishi-Arai Hospital, Tokyo, Japan
}

Nomura, K., Yamanouchi, T., Kim, G.U., Ohwaki, K. and Yano, E. Impact of Glucose Intolerance on Coronary Calcified Lesions Evaluated Using Multislice Computed Tomography. Tohoku J. Exp. Med., 2007, 212 (2), 107-113 — Metabolic syndrome has the unique concept that the common occurrence of individual disease components increases the risk of coronary artery disease (CAD). However, some studies suggest that the burden of different CAD risk factors is not equal, and focusing on the whole set of risk factors might neglect the impact of individual factors that could be useful targets for prophylactic therapies. The purpose of this study was to investigate the effect of glucose intolerance on CAD using multislice computed tomography (MSCT). Ninety-eight consecutive patients with at least one traditional CAD risk factor who visited a municipal hospital were enrolled in this study. The risk factors were impaired glucose tolerance (fasting glucose $\geqq 110 \mathrm{mg} /$ $\mathrm{dl}$ or patients with diabetes), low high-density lipoprotein cholesterol (HDL-C, $<40 \mathrm{mg} / \mathrm{dl}$ for men and $\leqq 50 \mathrm{mg} / \mathrm{dl}$ for women), hypertriglycemia (triglyceride $\geqq 150 \mathrm{mg} / \mathrm{dl}$ ), hypertension (blood pressure $\geqq 130 / 85 \mathrm{mmHg}$ ), and obesity (body mass index, $>25 \mathrm{~kg} / \mathrm{m}^{2}$ for men and $>23 \mathrm{~kg} / \mathrm{m}^{2}$ for women). CAD was determined by the presence of either stenoses, non-calcified plaques or calcified lesions. The following risk factors were significantly related in univariate logistic models: glucose intolerance and coronary calcified lesions ( $p$ $=0.001)$, and hypertriglycemia and non-calcified plaque lesions $(p=0.048)$. Multivariate models showed that glucose intolerance was significantly associated with calcified lesions, even after adjustment for gender, age, low HDL-C, hypertriglycemia, hypertension, and obesity ( $p=0.018$ ). Our results suggest that glucose intolerance might be closely related to the presence of coronary calcified lesions among traditional CAD risk factors.

coronary artery disease; coronary calcified lesion; glucose intolerance; metabolic syndrome; multislice computed tomography

(C) 2007 Tohoku University Medical Press

Coronary artery disease (CAD) is the leading cause of death in industrialized countries. Because CAD is often associated with sudden death from cardiac arrest, the early identification of high-risk subjects is critical. Therefore, risk factors for CAD have been studied extensively

Received February 5, 2007; revision accepted for publication April 16, 2007.

Correspondence: Kyoko Nomura, M.D., M.P.H., Ph.D., Department of Hygiene and Public Health, Teikyo

University, School of Medicine, Kaga 2-11-1, Itabashi, Tokyo 173-8605, Japan.

e-mail:kyoko@med.teikyo-u.ac.jp 
throughout the world. To date, several conditions, including hypertension, hypertriglycemia, hypercholesterolemia, hyperuricemia, diabetes mellitus, and obesity, have come to be considered significant risk factors (Lakka et al. 2002; Sargin et al. 2005; Tartan et al. 2006).

In 2001, the Third Adult Treatment Panel of the National Cholesterol Education Program introduced a new disease entity, namely, metabolic syndrome (Expert Panel on Detection 2001). This syndrome is a cluster of clinical characteristics that comprises a constellation of impaired glucose, insulin resistance, dyslipidemia, obesity, and hypertension; metabolic syndrome is diagnosed based on the presence of at least three of these five components.

Academics throughout the world have paid increasing attention to metabolic syndrome owing to the unique concept that the common occurrence of individual disease components increases the risk of CAD (Expert Panel on Detection 2001). Accordingly, a recent trend has been the emphasis of a set of risk factors for CAD presented by patients with metabolic syndrome, whereas other studies have underlined the impact of individual risk factors on CAD (Morici et al. 1997; Fisman et al. 2001; Anderson et al. 2004). For example, Anderson et al. (2004) investigated which features of metabolic syndrome predicted clinical outcomes of angiographic CAD and reported that glucose intolerance was the primary risk for CAD. If the burden of different CAD risk factors is not equal, focusing on the whole set of CAD risk factors might neglect the impact of individual risk factors that could be useful targets for the development of prophylactic therapies.

In previous studies that investigated the association between CAD risk factors and CAD using invasive coronary angiography, study subjects who needed to undergo angiography were likely to have advanced CAD. By contrast, multislice computed tomography (MSCT) permits noninvasive coronary assessment and therefore can be applied to a wide variety of test populations, regardless of CAD severity (Achenbach et al. 2000; Knez et al. 2000). However, one of the disadvantages of MSCT is the amount of radiation exposure; therefore, the indication for MSCT needs to be assessed before investigation. Referring to the American Heart Association scientific statement on the assessment of CAD using cardiac CT (Budoff et al. 2006), test populations should be at least at intermediate risk of cardiovascular disease, which is usually assessed by the Framingham 10-year risk estimate of coronary heart disease (CHD; Expert Panel on Detection 2001).

We sampled patients who were at intermediate risk of CHD and used MSCT to refine their coronary risk prediction. Our purpose was to determine if glucose intolerance predicts CAD equally to other traditional CAD risk factors, that is, to assess the impact of glucose intolerance on the development of CAD after adjustment for gender, age and other risk factors.

\section{Materials and Methods}

\section{Study design and patients}

This cross-sectional study was conducted using 107 consecutive patients with at least one CAD risk factor: obesity, hypertension, hyperlipidemia, or glucose intolerance, who visited the internal medicine clinic at a municipal hospital between January and May 2004. We excluded subjects with diabetes who were on insulin therapy, those who were unable to hold their breath for 30s (i.e., chronic lung disease), and those with unstable angina, cardiac arrhythmia, hemodynamic instability, or contraindications to the contrast agent (i.e., renal insufficiency, creatinine $>1.5 \mathrm{mg} / \mathrm{dl}$ ). Also, patients were excluded from the analysis if their coronary arteries could not be evaluated technically $(n=9)$. Hence, out study subjects for analyses became 98 patients (female, $61 \%$; mean age, 65 years) from whose medical records we retrospectively collected clinical biochemical data. In total, 73 patients (75\%) were on medication: 39, 42, and 34 patients received antihypertensive, antihyperlipidemic, and antidiabetic drug therapy. The detailed patient characteristics, including the Framingham 10-year risk scores, are shown (Table 1).

\section{Measurement of biochemical markers}

The biological and biochemical markers measured included gender, age, body mass index (BMI), systolic and diastolic blood pressures, fasting serum lipid concentrations (triglycerides and high-density lipoprotein cho- 
TABLE 1. Patient characteristics.

\begin{tabular}{|c|c|c|c|c|}
\hline & \multicolumn{2}{|c|}{$\begin{array}{c}\text { Men } \\
(n=37)\end{array}$} & \multicolumn{2}{|c|}{$\begin{array}{l}\text { Women } \\
(n=57)\end{array}$} \\
\hline & Median & $\mathrm{IQR}^{*}$ & Median & $\mathrm{IQR}^{*}$ \\
\hline Age (years) & 68 & $(60-72)$ & 65 & $(56-70)$ \\
\hline Systolic blood pressure (mmHg) & 139 & $(130-149)$ & 138 & $(125-156)$ \\
\hline Total cholesterol (mg/dl) & 201 & $(173-217)$ & 218 & $(188-247)$ \\
\hline HDL-C (mg/dl) & 51 & $(42-60)$ & 58 & $(48-76)$ \\
\hline Triglyceride (mg/dl) & 130 & $(93-225)$ & 121 & $(74-168)$ \\
\hline Fasting glucose (mg/dl) & 118 & $(97-147)$ & 98 & $(91-108)$ \\
\hline \multirow[t]{2}{*}{$\mathrm{BMI}\left(\mathrm{kg} / \mathrm{m}^{2}\right)^{\dagger}$} & 24 & $(21-27)$ & 24 & $(22-27)$ \\
\hline & $n$ & $\%$ & $n$ & $\%$ \\
\hline Current smoker ${ }^{\dagger}$ & 7 & 37 & 5 & 13 \\
\hline $\begin{array}{l}\text { Currently on any medication to treat high } \\
\text { blood pressure }\end{array}$ & 17 & 46 & 21 & 37 \\
\hline Framingham score (10-year risk) & \multicolumn{2}{|c|}{$12-30 \%$ and above } & \multicolumn{2}{|l|}{$4-11 \%$} \\
\hline
\end{tabular}

*Inter-quartile range (25-75\% of its distribution).

Number of missing data: BMI $=35$; current smokers $=40$.

The percentage was calculated based on the number of patients with systolic blood pressure $\geqq$ $130 \mathrm{mmHg}$ or diastolic blood pressure $\geqq 85 \mathrm{mmHg}$.

lesterol [HDL-C]), fasting plasma glucose concentrations, and hemoglobin A1c. The BMI was calculated as weight $(\mathrm{kg}) /$ height $\left(\mathrm{m}^{2}\right)$. Blood pressure was measured using a recently calibrated mercury sphygmomanometer on the right arm while the patient was seated after resting for at least $5 \mathrm{~min}$. Venous blood was collected after an overnight fast, and the serum was used for the biochemical tests. Serum triglyceride and HDL-C concentrations were measured enzymatically (JCA-HR2200; Japan Electron Optics Laboratory, Tokyo). The glucose concentration was measured using an enzymatic hexokinase method. Hemoglobin A1c was measured by immunoassay using monoclonal antibodies (DM-JACK; Kyowa Medex, Tokyo). Quality control was assessed by comparison with suitable reference materials and was depicted on a quality control chart.

\section{Risk factors for CAD}

With reference to the metabolic syndrome introduced by the Third Adult Treatment Panel of the National Cholesterol Education Program (Expert Panel on Detection 2001), the CAD risk factors that we assessed were obesity, hypertension, low HDL-C concentration, hypertriglycemia, and glucose intolerance. A subject was defined as: (1) obese if the BMI was $>25 \mathrm{~kg} / \mathrm{m}^{2}$ for men and $>23 \mathrm{~kg} / \mathrm{m}^{2}$ for women; (2) hypertensive if the blood pressure was $\geqq 130 / 85 \mathrm{mmHg}$ or if the patient was undergoing antihypertensive therapy; (3) having a low HDL-C concentration if the HDL-C was $<40 \mathrm{mg} / \mathrm{dl}$ for men or $\leqq 50 \mathrm{mg} / \mathrm{dl}$ for women; (4) hypertriglycemic if the serum triglyceride concentration was $\geqq 150 \mathrm{mg} / \mathrm{dl}$ or if the patient was on lipid-lowering drugs; (5) glucose intolerant if the serum fasting glucose concentration was $\geqq 110 \mathrm{mg} / \mathrm{dl}$ or the $\mathrm{HbA} 1 \mathrm{c}$ was $>6.2 \mathrm{~g} / \mathrm{dl}$, or if the patient was clinically diagnosed with diabetes.

\section{MSCT protocol}

All patients with a heart rate $>60 \mathrm{bpm}$ received 50 $\mathrm{mg}$ atenolol (Tenormin; AstraZeneca, Wilmington, DE) orally $1 \mathrm{hr}$ before the MSCT scan. The images were obtained with a 16-slice MSCT scanner (Aquilion 16 Super Heart; Toshiba, Tochigi) using contrast-enhanced spiral scanning and retrospective electrocardiography (ECG) gating.

The following parameters were used for angiographic scanning: slice collimation, $16 \times 0.5 \mathrm{~mm}$; gantry rotation time, $0.4 \mathrm{~s} /$ rotation; tube current and voltage, $400 \mathrm{~mA}$ and $120 \mathrm{kV}$, respectively. For the contrastenhanced scan, $50 \mathrm{ml}$ of nonionic iodinated contrast agent (Omnipaque350; Daiichi Pharmaceutical, Tokyo) was injected at $3.0 \mathrm{ml} / \mathrm{s}$, followed by $30 \mathrm{ml}$ at $1.5 \mathrm{ml} / \mathrm{s}$. The start of contrast-enhanced scanning was adapted to 
bolus tracking system images (Kirchner 2000). All scans were performed during a single breath-hold. Scanning was extended from the distal segment of the left anterior descending coronary artery to cover the whole heart. The raw data of the scans were reconstructed using algorithms optimized for retrograde ECG-gated multislice spiral reconstruction. Data sets were reconstructed in the cardiac cycle $(0,10,20,30,40,50,60,70,80$, and $90 \%$ of the R-R interval), and those with the fewest motion artifacts were used for further evaluation of the coronary artery.

With collimation 16-slice $\times 0.5 \mathrm{~mm}, 0.4 \mathrm{~s} /$ rotation, detector pitch 3.2 , and $100-\mathrm{mm}$ range, the weighted computed tomography dose index (CTDIw) and dose length product (DLP) were $98.5 \mathrm{mGy}$ and $1.05 \mathrm{~Gy} \mathrm{~cm}$, respectively.

\section{Image evaluation}

The reconstructed image data were transferred to a computer workstation for post-processing (ZIO M900; Amin/ZIO, Tokyo). Axial images, curved multiplanar reconstructions along and orthogonal to the vessel, threedimensional (3D) reconstructions, and maximum intensity projections were used to detect stenoses in the four main coronary branches (left main coronary artery; left anterior descending coronary artery; left circumflex coronary artery; and right coronary artery). Stenoses were defined as significant if the luminal reduction was $\geqq$ $50 \%$. For non-calcified plaque detection, cross-sectional and curved multiplanar reformation images were analyzed. Any calcified lesions were also recorded. Two reviewers (a cardiologist and a radiologist), who were blind to the biochemical data, analyzed the MSCT data independently. In cases of disagreement, the images were reviewed until a consensus was reached.

\section{Data analyses}

A logistic regression model was used to assess the impact of five CAD risk factors on CAD defined by coronary stenoses, non-calcified plaques, and calcified lesions. Hence, three models with three different CAD outcomes were used. Crude (univariate) and adjusted (multivariate) odds ratios (OR) were calculated with 95\% confidence intervals $(95 \% \mathrm{CI})$. Due to the relatively large number of missing data for BMI, two multivariate models were used to assess the impact of glucose intolerance on the development of CAD: Model I adjusted for gender, age, hypertension, low HDL-C concentration, and hypertriglycemia; Model II adjusted for the explanatory variables in Model I and obesity. Age was treated as a continuous variable in the logistic regression model because the presence of calcified lesions appeared to increase proportionately with age. The OR reflects an increase in the odds of CAD lesions for each explanatory variable. The analyses were carried out using SAS version 8.12 for Windows (SAS, Cary, NC, USA).

\section{Results}

Glucose intolerance was observed in 48 patients: those with impaired fasting glucose $(n=$ 23) and those with clinical diabetes $(n=25)$. Patient characteristics, including concomitant rates of other CAD risk factors, were similar between patients with glucose intolerance and those without. Patients were tabulated according to the five CAD risk factors, and the proportions of CAD defined by coronary stenoses, non-calcified plaques, and calcified lesions were determined (Table 2). Among the CAD risk factors, stenoses were most frequently observed in patients with

TABLE 2. The proportion of patients with CAD in patients with each CAD risk factor.

\begin{tabular}{lcccccc}
\hline \multirow{2}{*}{ CAD risk factor } & \multicolumn{2}{c}{ Total $(n=98)$} & & \multicolumn{3}{c}{ CAD } \\
\cline { 2 - 3 } \cline { 6 - 7 } & $n$ & $\%$ & & Stenoses $(\%)$ & $\begin{array}{c}\text { Non-calcified } \\
\text { plaques }(\%)\end{array}$ & $\begin{array}{c}\text { Calcified lesions } \\
(\%)\end{array}$ \\
\hline Obesity* $^{*}$ & 38 & 66 & & 32 & 18 & 45 \\
Hypertension & 76 & 78 & & 38 & 17 & 46 \\
Low HDL-C & 55 & 56 & & 36 & 15 & 49 \\
Hypertriglycemia & 59 & 60 & & 31 & 25 & 44 \\
Glucose intolerance & 48 & 49 & & 35 & 25 & 58 \\
\hline
\end{tabular}

The definition of each CAD risk factor is described in the text.

*Number of missing data $=35$. 
hypertension, non-calcified plaques were most frequently observed in patients with hypertriglycemia or glucose intolerance, and calcified lesions were most frequently observed in patients with glucose intolerance.

Univariate logistic models showed that the significant CAD risk factors were hypertriglycemia in the model with the outcome of non-calcified plaque lesions, and glucose intolerance in the model of calcified lesions. No association was observed between any of the CAD risk factors and coronary stenoses. Multivariate analyses showed that among three types of CAD lesions, only coronary calcified lesions were significantly associated with CAD risk factors (Table 3). After adjusting for gender, age, hypertension, low HDL-C concentration, and hypertriglycemia, Model I showed that significant risk factors for calcified lesions were glucose intolerance (OR, 5.92; 95\% CI, 1.84-19.00), age (OR, 1.11; 95\% CI, 1.04-1.20), and low HDL-C concentration (OR, 3.30; 95\% CI, 1.07-10.18). However, in Model II, the effect of low HDL-C disappeared, and glucose intolerance (OR, 7.31; 95\% CI, 1.41-38.0) and age (OR, 1.18; 95\% CI, 1.05-1.30) became the only independent and significant risk factors for calcified lesions. There was no significant interaction between any of the possible pairs of risk factors and calcified lesions in either of the models.

\section{Discussion}

We investigated the association between CAD, defined by three different characteristics of coronary artery lesions and the five traditional CAD risk factors, and demonstrated that glucose intolerance was significantly associated with calcified coronary lesions after adjusting for gender, age, and other CAD risk factors. Our results suggest that the individual risk of CAD risk factors might not be equal; therefore, preventive measures may be needed based on the individual risk of each factor. The interpretation of these results is discussed below in light of the limitations and strengths of the study.

Among models with three CAD outcomes, the significant and consistent finding in both univariate and multivariate analyses was the effect of glucose intolerance on coronary calcified lesions only; there were no such associations between CAD risk factors and stenoses or non-calcified plaques in multivariate analyses. The discrepancy among the three models with three different CAD outcomes might be accounted for by the diagnostic performance of the 16-row MSCT used. Although the efficacy of the 16-row MSCT has

TABLE 3. Odds ratios (ORs) with $95 \%$ confidence intervals (CIs) for the impact of CAD risk factors on coronary calcified lesions.

\begin{tabular}{|c|c|c|c|c|c|c|c|c|c|c|c|}
\hline \multirow{4}{*}{$\mathrm{CAD}$ risk factor } & & & & & & \multicolumn{6}{|c|}{ Multivariate $^{\dagger}$} \\
\hline & \multirow{2}{*}{\multicolumn{2}{|c|}{$\begin{array}{c}\text { Coronary } \\
\text { Calcified lesions }\end{array}$}} & \multicolumn{3}{|c|}{ Univariate } & \multicolumn{3}{|c|}{ Model I $(n=98)$} & \multicolumn{3}{|c|}{ Model II $(n=63)$} \\
\hline & & & \multirow[t]{2}{*}{ OR } & \multicolumn{2}{|c|}{$95 \% \mathrm{CI}^{*}$} & \multirow[t]{2}{*}{ OR } & \multicolumn{2}{|c|}{$95 \% \mathrm{CI}^{*}$} & \multirow[t]{2}{*}{ OR } & \multicolumn{2}{|c|}{$95 \% \mathrm{CI}^{*}$} \\
\hline & $n$ & $\%$ & & Lower & Upper & & Lower & Upper & & Lower & Upper \\
\hline Obesity & 17 & 45 & 0.65 & 0.22 & 1.92 & - & - & - & 0.90 & 0.16 & 4.95 \\
\hline Hypertension & 35 & 46 & 2.55 & 0.88 & 7.44 & 2.12 & 0.52 & 8.69 & 0.55 & 0.09 & 3.59 \\
\hline Low HDL-C & 27 & 49 & 2.30 & 0.96 & 5.47 & 3.30 & 1.07 & 10.18 & 2.94 & 0.67 & 12.94 \\
\hline Hypertriglycemia & 26 & 44 & 1.33 & 0.56 & 3.16 & 1.11 & 0.35 & 3.53 & 1.43 & 0.28 & 7.21 \\
\hline Glucose intolerance & 28 & 58 & 4.45 & 1.81 & 10.97 & 5.92 & 1.84 & 19.00 & 7.31 & 1.41 & 37.99 \\
\hline
\end{tabular}

The definition of each CAD risk factor is described in the text.

*95\% CIs are based on the Wald Chi-square test.

Model I was adjusted for gender, age, and the presence of CAD risk factors; Model II was adjusted for the explanatory variables used in Model I and BMI.

Number of missing data $=35$. 
been considered controversial, two recent important studies (Hoffmann et al. 2004; Garcia et al. 2006) have reached the conclusion that MSCT coronary angiography performed using 16-row scanners is limited by the high number of nonevaluable cases and the high rate of false-positives due to coronary calcification. Hence, the misclassification of coronary stenoses and non-calcified plaque lesions may have reduced the statistical significance of their models.

In support of this, within the accumulated body of evidence that suggests a proportional relationship between CHD risk and the extent of coronary artery calcium (CAC; Wong et al. 2003, 2005; Anand et al. 2006), Mitsutake et al. (2006) investigated the diagnostic accuracy of coronary artery calcification measured using 16-row MSCT compared with that of invasive coronary angiography. The CAC score determined using 16-row MSCT could predict CAD independent of other factors such as age, metabolic disease, and medication, suggesting the usefulness of CT, especially when coronary stenosis cannot be diagnosed because of severe calcification.

There is accumulating evidence that glucose intolerance is an independent risk factor for cardiovascular disease. A few epidemiological studies (Seibaek et al. 1997; Wong et al. 2003; Kataoka et al. 2005) have suggested that abnormal glucose metabolism plays an important role in the development of CHD, suggesting a positive relationship between insulin resistance and CAD. Muis et al. (2005) reported that coronary vascular damage was more severe in diabetes mellitus (DM) patients who were taking insulin treatment than in those who were not. We excluded DM patients who were taking insulin therapy; therefore, our patients were likely composed of DM patients without severe CAD and patients with impaired fasting glucose with and without DM. Thus, our results support the notion that glucose intolerance, indicating insulin resistance, contributes to the development of CAD, regardless of its severity.

Because of the small sample size for BMI, the $95 \%$ CI for glucose intolerance in Model II were relatively wide. Nevertheless, the variables in Model II were metabolic syndrome constituents, and our results suggest that among the five risk factors studied, i.e., obesity, hypertension, low HDL-C concentration, hypertriglycemia, and glucose intolerance, only glucose intolerance was closely related to coronary calcified lesions. This may indicate that individual CAD risk factors do not contribute equally to the development of CAD. Focusing on individual CAD risk factors, rather than a set of such factors, i.e., metabolic syndrome disease, may facilitate the development of prophylactic strategies against the development of CAD.

\section{Acknowledgments}

We thank Professor Shigeru Furui and Dr. Shigeru Suzuki at Department of Radiology, Teikyo University School of Medicine for their valuable comments on this manuscript and Mss. Kaori Chiba at Nishiarai Hospital for her assistance with the data collection.

\section{Reference}

Achenbach, S., Ulzheimer, S., Baum, U., Kachelriess, M., Ropers, D., Giesler, T., Bautz, W., Daniel, W.G., Kalender, W.A. \& Moshage, W. (2000) Noninvasive coronary angiography by retrospectively ECG-gated multislice spiral CT. Circulation, 102, 2823-2828.

Anand, D.V., Lim, E., Hopkins, D., Corder, R., Shaw, L.J., Sharp, P., Lipkin, D. \& Lahiri, A. (2006) Risk stratification in uncomplicated type 2 diabetes: prospective evaluation of the combined use of coronary artery calcium imaging and selective myocardial perfusion scintigraphy. Eur. Heart J., 27, 713-721.

Anderson, J.L., Horne, B.D., Jones, H.U., Reyna, S.P., Carlquist, J.F., Bair, T.L., Pearson, R.R., Lappe, D.L. \& Muhlestein, J.B. (2004) Which features of the metabolic syndrome predict the prevalence and clinical outcomes of angiographic coronary artery disease? Cardiology, 101, 185-193.

Budoff, M.J., Achenbach, S., Blumenthal, R.S., Carr, J.J., Goldin, J.G., Greenland, P., Guerci, A.D., Lima, J.A., Rader, D.J., Rubin, G.D. \& Shaw, L.J. (2006) Assessment of coronary artery disease by cardiac computed tomography: a scientific statement from the American Heart Association Committee on cardiovascular imaging and intervention, council on cardiovascular radiology and intervention, and committee on cardiac imaging, council on clinical cardiology. Circulation, 114, 1761-1791.

Expert Panel on Detection, Evaluation, and Treatment of High Blood Cholesterol in Adults (2001) Executive summary of the third report of the national cholesterol education program (NCEP) expert panel on detection, evaluation, and treatment of high blood cholesterol in adults (Adult Treatment Panel III). JAMA, 285, 2486-2497.

Fisman, E.Z., Motro, M., Tenenbaum, A., Boyko, V., Mandelzweig, L. \& Behar, S. (2001) Impaired fasting 
glucose concentrations in nondiabetic patients with ischemic heart disease: a marker for a worse prognosis. Am. Heart J., 141, 485-490.

Garcia, M.J., Lessick, J. \& Hoffmann, M.H. (2006) Accuracy of 16-row multidetector computed tomography for the assessment of coronary artery stenosis. JAMA, 296, 403-411.

Hoffmann, U., Moselewski, F., Cury, R.C., Ferencik, M., Jang, I.K., Diaz, L.J., Abbara, S., Brady, T.J. \& Achenbach, S. (2004) Predictive value of 16-slice multidetector spiral computed tomography to detect significant obstructive coronary artery disease in patients at high risk for coronary artery disease: patient-versus segment-based analysis. Circulation, 110, 2638-2643.

Kataoka, Y., Yasuda, S., Morii, I., Otsuka, Y., Kawamura, A. \& Miyazaki, S. (2005) Quantitative coronary angiographic studies of patients with angina pectoris and impaired glucose tolerance. Diabetes Care, 28, 2217-2222.

Kirchner, J., Kickuth, R., Laufer, U., Noack, M. \& Liermann, D. (2000) Optimized enhancement in helical CT: experiences with a real-time bolus tracking system in 628 patients. Clin. Radiol., 55, 368-373.

Knez, A., Becker, C., Ohnesorge, B., Haberl, R., Reiser, M. \& Steinbeck, G. (2000) Noninvasive detection of coronary artery stenosis by multislice helical computed tomography. Circulation, 101, E221-E222.

Lakka, H.M., Laaksonen, D.E., Lakka, T.A., Niskanen, L.K., Kumpusalo, E., Tuomilehto, J. \& Salonen, J.T. (2002) The metabolic syndrome and total and cardiovascular disease mortality in middle-aged men. JAMA, 288, 2709-2716.

Mitsutake, R., Niimura, H., Miura, S., Zhang, B., Iwata, A., Nishikawa, H., Kawamura, A., Kumagai, K., Shirai, K., Matsunaga, A. \& Saku, K. (2006) Clinical significance of the coronary calcification score by multidetector row computed tomography for the evaluation of coronary stenosis in Japanese patients. Circ. J., 70, 1122-1127.

Morici, M.L., Di Marco, A., Sestito, D., Candore, R., Cangemi, C., Accardo, F., Donatelli, M., Cataldo, M.G. \& Lombardo, A. (1997) The impact of coexistent diabetes on the prevalence of coronary heart disease. J. Diabetes Complications, 11, 268-273.

Muis, M.J., Bots, M.L., Bilo, H.J., Hoogma, R.P., Hoekstra, J.B., Grobbee, D.E. \& Stolk, R.P. (2005) High cumulative insulin exposure: a risk factor of atherosclerosis in type 1 diabetes? Atherosclerosis, 181, 185-192.

Sargin, H., Ozisik, M., Ozisik, N.C., Seven, O., Orbay, E., Gozu, H., Sargin, M., Tekce, M. \& Yayla, A. (2005) The prevalence of silent ischemia in Turkish patients with type 2 diabetes mellitus. Tohoku J. Exp. Med., 205, 351-355.

Seibaek, M., Sloth, C., Vallebo, L., Hansen, T., Urhammer, S.A., Burchardt, H., Torp-Pedersen, C., Pedersen, O. \& Hildebrandt, P. (1997) Glucose tolerance status and severity of coronary artery disease in men referred to coronary arteriography. Am. Heart J., 133, 622-629.

Tartan, Z., Uyarel, H., Kasikcioglu, H., Alper, A.T., Ozay, B., Bilsel, T., Gul, M., Ozturk, R. \& Cam, N. (2006) Metabolic syndrome as a predictor of non-dipping hypertension. Tohoku J. Exp. Med., 210, 57-66.

Wong, N.D., Sciammarella, M.G., Polk, D., Gallagher, A., Miranda-Peats, L., Whitcomb, B., Hachamovitch, R., Friedman, J.D., Hayes, S. \& Berman, D.S. (2003) The metabolic syndrome, diabetes, and subclinical atherosclerosis assessed by coronary calcium. J. Am. Coll. Cardiol., 41, 1547-1553.

Wong, N.D., Rozanski, A., Gransar, H., Miranda-Peats, R., Kang, X., Hayes, S., Shaw, L., Friedman, J., Polk, D. \& Berman, D. (2005) Metabolic syndrome and diabetes are associated with an increased likelihood of inducible myocardial ischemia among patients with subclinical atherosclerosis. Diabetes Care, 28, 1445-1450. 